\title{
Generalization of Certain Subclasses of Multivalent Functions with Negative Coefficients Defined by Cho-Kwon-Srivastava Operator
}

\author{
Elsayed A. Elrifai, Hanan E. Darwish, Abdusalam R. Ahmed \\ Department of Mathematics, Faculty of Science, University of \\ Mansoura, Mansoura, Egypt \\ E-mail:Rifai@mans.edu.eg,Darwish333@yahoo.com,Abdusalam5056@yahoo.com \\ Received November 1, 2010; revised July 6, 2011; accepted July 13, 2011
}

\begin{abstract}
Making use of the Cho-Kwon-Srivastava operator, we introduce and study a certain $S C_{n}(j, p, \lambda, \alpha, \delta)$ of $p$-valently analytic functions with negative coefficients. In this paper, we obtain coefficient estimates, distortion theorem, radii of close-to-convexity, starlikeness, convexity and modified Hadamard products of functions belonging to the class $S C_{n}(j, p, \lambda, \alpha, \delta)$. Finally, several applications investigate an integral operator, and certain fractional calculus operators also considered.
\end{abstract}

Keywords: Multivalent Functions, Cho-Kwon-Srivastava Operator, Modified-Hadamard Product, Fractional Calculus

\section{Introduction}

Let $T(j, p)$ denote the class of functions of the form:

$$
f(z)=z^{p}-\sum_{k=j+p}^{\infty} a_{k} z^{k}\left(a_{k} \geq 0 ; p, j \in N=\{1,2,3, \cdots\}\right),
$$

which are analytic and p-valent in the open unit disc $U=\{z:|z|<1\}$. A function $f(z) \in T(j, p)$ is said to be $p$-valently starlike of order $\alpha$ if it satisfies the inequality :

$$
\operatorname{Re}\left(\frac{z f^{\prime}(z)}{f(z)}\right)>\alpha(z \in U ; 0 \leq \alpha<p ; p \in N) .
$$

We denote by $T_{j}^{*}(p, \alpha)$ the class of all $p$-valently starlike functions of order $\alpha$. Also a function $f(z) \in$ $T(j, p)$ is said to be $p$-valently convex of order $\alpha$ if it satisfies the inequality:

$$
\operatorname{Re}\left\{1+\frac{z f^{\prime \prime}(z)}{f^{\prime}(z)}\right\}>\alpha(z \in U ; 0 \leq \alpha<p ; p \in N) \text {. }
$$

We denote by $C_{j}(p, \alpha)$ the class of all $p$-valently convex functions of order $\alpha$. We note that (see for example Duren [1] and Goodman [2])

$$
f(z) \in C_{j}(p, \alpha) \Leftrightarrow \frac{z f^{\prime}(z)}{p} \in T_{j}^{*}(p, \alpha)
$$

$$
(0 \leq \alpha<p ; p \in N) \text {. }
$$

The classes $T_{j}^{*}(p, \alpha)$ and $C_{j}(p, \alpha)$ are studied by Owa [3].

In [4] Wang et al. defined Cho-kwon-Srivastava operator which

$$
\ell_{p, j}^{\lambda}(a, c) f(z): T(j, p) \rightarrow T(j, p),
$$

by

$$
\ell_{p, j}^{\lambda}(a, c) f(z)=z^{p}-\sum_{k=j+p}^{\infty} \frac{(\lambda+p)_{k-p}(c)_{k-p}}{(k-p) !(a)_{k-p}} a_{k} z^{k}
$$

for

$$
\left(a, c \in R / Z_{0}^{-}=\{0,-1,-2,-3, \cdots\}, z \in U, \lambda>-p\right)
$$

and

$$
(\lambda)_{k}= \begin{cases}1 & ; k=0 \\ \lambda(\lambda+1) \cdots(\lambda+k-1) & ; k \in N .\end{cases}
$$

Clearly, $\ell_{p, 1}^{\lambda}(a, c)$ is the well-known Cho-kwonSrivastava operator (see [5]) where

$$
\ell_{p, j}^{1}(p+1,1)=f(z), \ell_{p, j}^{1}(p, 1)=\frac{z f^{\prime}(z)}{p},
$$


and

$$
\ell_{p, 1}^{\lambda}(a, a)=D^{\lambda+p-1} f(z)(\lambda>-p),
$$

where $D^{\lambda+p-1}$ is the well-known Ruscheweyh derivative of $(\lambda+p-1)$-th order.

With the help of the Cho-Kwon-Srivastava $\ell_{p, j}^{\lambda}(a, c) f(z)$, we say that a function $f(z)$ belonging to $T(j, p)$ is in the class $S C_{n}(j, p, \lambda, \alpha, \delta)$ if and only if

$$
\operatorname{Re}\left\{\frac{z\left(\ell_{p, j}^{\lambda}(a, c) f(z)\right)^{\prime}+\delta z^{2}\left(\ell_{p, j}^{\lambda}(a, c) f(z)\right)^{\prime \prime}}{(1-\delta) \ell_{p, j}^{\lambda}(a, c) f(z)+\delta z\left(\ell_{p, j}^{\lambda}(a, c) f(z)\right)^{\prime}}\right\}>\alpha
$$

We note that:

1) when $\delta=0$, we have

$$
\operatorname{Re}\left\{\frac{z\left(\ell_{p, j}^{\lambda}(a, c) f(z)\right)^{\prime}}{\ell_{p, j}^{\lambda}(a, c) f(z)}\right\}>\alpha
$$

which is the class of starlike of order $\alpha$.

2) when $\delta=0, a=p+1, \lambda=1, c=1$, we have the class

$$
\operatorname{Re}\left\{\frac{z f^{\prime}(z)}{f(z)}\right\}>\alpha ; 0 \leq \alpha<p
$$

which is the class of starlike functions of order $\alpha$ studied by Owa [3] and Yamakawa [6]

3) when $\delta=1$, we have

$$
\operatorname{Re}\left\{1+\frac{z\left(\ell_{p, j}^{\lambda}(a, c) f(z)\right)^{\prime \prime}}{\left(\ell_{p, j}^{\lambda}(a, c) f(z)\right)^{\prime}}\right\}>\alpha ;
$$

which is the class of convex operator of order $\alpha$.

4) when $\delta=1, a=p+1, c=1, \lambda=1$, we have

$$
\operatorname{Re}\left\{1+\frac{z f^{\prime \prime}(z)}{f^{\prime}(z)}\right\}>\alpha ;(0 \leq \alpha<p)
$$

which is the class of convex functions of order $\alpha$ studied by Owa [3] and Yamakawa [6].

In our present paper, we shall make use of the familiar $J_{c, p}$ defined by (c.f. [7,8], see also [9])

$$
\begin{gathered}
\left(J_{c, p} f\right)(z)=\frac{c+p}{z^{c}} \int_{0}^{z} t^{c-1} f(t) \mathrm{d} t, \\
(f(z) \in T(j, p) ; c>-p, p \in N),
\end{gathered}
$$

as well as the fractional calculus operator $D_{z}^{\mu}$ for which it is well known that (see, for details, $[10,11]$; see also Section 5 below)

$$
\begin{gathered}
D_{z}^{\mu}\left\{z^{\rho}\right\}=\frac{\Gamma(\rho+1)}{\Gamma(\rho+1-\mu)} z^{\rho-\mu}, \\
(\rho>-1 ; \mu \in R)
\end{gathered}
$$

in terms of Gamma functions.

\section{Coefficient Estimates}

Theorem 1. Let the function $f(z)$ defined by (1.1). Then $f(z) \in S C_{n}(j, p, \lambda, \alpha, \delta)$ if and only if

$$
\begin{gathered}
\sum_{k=j+p}^{\infty} \frac{(\lambda+p)_{k-p}(c)_{k-p}}{(k-p) !(a)_{k-p}}(k-\alpha)[1+\delta(k-1)] a_{k} \\
\leq(p-\alpha)[1+\delta(p-1)] \\
\left(z \in U ; 0 \leq \alpha<p, 0 \leq \delta \leq 1, p, j \in N, n \in N_{0}\right) .
\end{gathered}
$$

Proof. Assume that the inequality (2.1) holds true. Then we have

$$
\begin{aligned}
(j+p-\alpha) & \sum_{k=j+p}^{\infty} \frac{(\lambda+p)_{k-p}(c)_{k-p}}{(k-p) !(a)_{k-p}}[1+\delta(k-1)] a_{k} \\
& \leq \sum_{k=j+p}^{\infty} \frac{(\lambda+p)_{k-p}(c)_{k-p}}{(k-p) !(a)_{k-p}}(k-\alpha)[1+\delta(k-1)] a_{k} \leq(p-\alpha)[1+\delta(p-1)]
\end{aligned}
$$

that is, that

$$
\sum_{k=j+p}^{\infty} \frac{(\lambda+p)_{k-p}(c)_{k-p}}{(k-p) !(a)_{k-p}}[1+\delta(k-1)] a_{k} \leq \frac{(p-\alpha)[1+\delta(p-1)]}{j+p-\alpha}
$$

Since 


$$
\begin{aligned}
& \left|[1+\delta(p-1)]-\sum_{k=j+p}^{\infty} \frac{(\lambda+p)_{k-p}(c)_{k-p}}{(k-p) !(a)_{k-p}}[1+\delta(k-1)] a_{k} z^{k-p}\right| \\
& \geq[1+\delta(p-1)]-\sum_{k=j+p}^{\infty} \frac{(\lambda+p)_{k-p}(c)_{k-p}}{(k-p) !(a)_{k-p}}[1+\delta(k-1)] a_{k}|z|^{k-p} \\
& \geq[1+\delta(p-1)]-\sum_{k=j+p}^{\infty} \frac{(\lambda+p)_{k-p}(c)_{k-p}}{(k-p) !(a)_{k-p}}[1+\delta(k-1)] a_{k} \geq \frac{j[1+\delta(p-1)]}{j+p-\alpha}>0 .
\end{aligned}
$$

Then we find that

$$
\begin{aligned}
& \left|\frac{z\left(\ell_{p, j}^{\lambda}(a, c) f(z)\right)^{\prime}+\delta z^{2}\left(\ell_{p, j}^{\lambda}(a, c) f(z)\right)^{\prime \prime}}{(1-\delta) \ell_{p, j}^{\lambda}(a, c) f(z)+\delta z\left(\ell_{p, j}^{\lambda}(a, c) f(z)\right)^{\prime}}-p\right| \\
& \leq \frac{\sum_{k=j+p}^{\infty} \frac{(\lambda+p)_{k-p}(c)_{k-p}}{(k-p) !(a)_{k-p}}(k-p)[1+\delta(k-1)] a_{k}|z|^{k-p}}{[1+\delta(k-1)]-\sum_{k=j+p}^{\infty} \frac{(\lambda+p)_{k-p}(c)_{k-p}}{(k-p) !(a)_{k-p}}[1+\delta(k-1)] a_{k}|z|^{k-p}} \\
& \leq \frac{\sum_{k=j+p}^{\infty} \frac{(\lambda+p)_{k-p}(c)_{k-p}}{(k-p) !(a)_{k-p}}(k-p)[1+\delta(k-1)] a_{k}}{[1+\delta(p-1)]-\sum_{k=j+p}^{\infty} \frac{(\lambda+p)_{k-p}(c)_{k-p}}{(k-p) !(a)_{k-p}}[1+\delta(k-1)] a_{k}} \leq p-\alpha .
\end{aligned}
$$

This shows that the values of the function

$$
\phi(z)=\frac{z\left(\ell_{p, j}^{\lambda}(a, c) f(z)\right)^{\prime}+\delta z^{2}\left(\ell_{p, j}^{\lambda}(a, c) f(z)\right)^{\prime \prime}}{(1-\delta) \ell_{p, j}^{\lambda}(a, c) f(z)+\delta z\left(\ell_{p, j}^{\lambda}(a, c) f(z)\right)^{\prime}}
$$

lie in a circle which is centered at $w=p$ and whose radius is $(p-\alpha)$. Hence $f(z)$ satisfies the condition (1.6).

Conversely, assume that the function $f(z)$ is in the class $S C_{n}(j, p, \lambda, \alpha, \delta)$. Then we have

$$
\begin{aligned}
& \operatorname{Re}\left\{\frac{z\left(\ell_{p, j}^{\lambda}(a, c) f(z)\right)^{\prime}+\delta z^{2}\left(\ell_{p, j}^{\lambda}(a, c) f(z)\right)^{\prime \prime}}{(1-\delta) \ell_{p, j}^{\lambda}(a, c) f(z)+\delta z\left(\ell_{p, j}^{\lambda}(a, c) f(z)\right)^{\prime}}\right\} \\
& =\operatorname{Re}\left\{\frac{p[1+\delta(p-1)]-\sum_{k=j+p}^{\infty} \frac{(\lambda+p)_{k-p}(c)_{k-p}}{(k-p) !(a)_{k-p}} k[1+\delta(k-1)] a_{k} z^{k-p}}{[1+\delta(p-1)]-\sum_{k=j+p}^{\infty} \frac{(\lambda+p)_{k-p}(c)_{k-p}}{(k-p) !(a)_{k-p}}[1+\delta(k-1)] a_{k} z^{k-p}}\right\}>\alpha
\end{aligned}
$$

for some $\alpha(0 \leq \alpha<p)$, some $\delta(0 \leq \delta \leq 1), p, \quad j \in N$, $n \in N_{0}$, and $z \in U$. Choose values of $z$ on the real axis denominator in (2.4) and letting $z \rightarrow 1^{-}$through real so that $\phi(z)$ given by (2.3) is real. Upon clearing the values, we can see that

$$
\begin{aligned}
& p[1+\delta(p-1)]-\sum_{k=j+p}^{\infty} \frac{(\lambda+p)_{k-p}(c)_{k-p}}{(k-p) !(a)_{k-p}} k[1+\delta(k-1)] a_{k} \\
& \quad \geq \alpha\left\{[1+\delta(p-1)]-\sum_{k=j+p}^{\infty} \frac{(\lambda+p)_{k-p}(c)_{k-p}}{(k-p) !(a)_{k-p}}[1+\delta(k-1)] a_{k}\right\} .
\end{aligned}
$$


Thus we have the inequality (2.1).

Corollary 1. Let the function $f(z)$ defined by (1.1) be in the class $S C_{n}(j, p, \lambda, \alpha, \delta)$. Then

$$
\begin{gathered}
a_{k} \leq \frac{(p-\alpha)[1+\delta(p-1)]}{\frac{(\lambda+p)_{k-p}(c)_{k-p}}{(k-p) !(a)_{k-p}}(k-\alpha)[1+\delta(k-1)]} \\
(k \geq j+p, p, j \in N) .
\end{gathered}
$$

The result is sharp for the function $f(z)$ given by

$$
\begin{gathered}
f(z)=z^{p}-\frac{(p-\alpha)[1+\delta(p-1)]}{\frac{(\lambda+p)_{k-p}(c)_{k-p}}{(k-p) !(a)_{k-p}}(k-\alpha)[1+\delta(k-1)]} z^{k} \\
\left(k \geq j+p, p, j \in N, n \in N_{0}\right) .
\end{gathered}
$$

\section{Distortion Theorem}

Theorem 2. If a function $f(z)$ defined by (1.1) is in the class $S C_{n}(j, p, \lambda, \alpha, \delta)$ then

$$
\begin{aligned}
& \left\{\frac{p !}{(p-m) !}-\frac{(p-\alpha)[1+\delta(p-1)](j+p) !}{\frac{(\lambda+p)_{j}(c)_{j}}{j !(a)_{j}}(j+p-\alpha)[1+\delta(j+p-1)](j+p-m) !}|z|^{j}\right\}|z|^{p-m} \\
& \leq\left|f^{m}(z)\right| \leq\left\{\frac{p !}{(p-m) !}+\frac{(p-\alpha)[1+\delta(p-1)](j+p) !}{\frac{\left.(\lambda+p)_{j}(c)\right)_{j}}{j !(a)_{j}}(j+p-\alpha)[1+\delta(j+p-1)](j+p-m) !}|z|^{j}\right\}|z|^{p-m} \\
& \left(z \in U ; 0 \leq \alpha<p ; 0 \leq \lambda \leq 1, p, j \in N, n \in N_{0}\right) .
\end{aligned}
$$

The result is sharp for the function $f(z)$ given by

$$
f(z)=z^{p}-\frac{(p-\alpha)[1+\delta(p-1)]}{\frac{(\lambda+p)_{j}(c)_{j}}{j !(a)_{j}}(j+p-\alpha)[1+\delta(j+p-1)]} z^{j+p} \cdot\left(p, j \in N, n \in N_{0}\right) .
$$

Proof. In view of Theorem 1, we have

$$
\frac{\left(\frac{(\lambda+p)_{j}(c)_{j}}{j !(a)_{j}}\right)(j+p-\alpha)[1+\delta(j+p-1)]}{(p-\alpha)[1+\delta(p-1)](j+p) !} \sum_{k=j+p}^{\infty} k ! a_{k} \leq \sum_{k=j+p}^{\infty} \frac{\frac{(\lambda+p)_{k-p}(c)_{k-p}}{(k-p) !(a)_{k-p}}(k-\alpha)[1+\delta(k-1)]}{(p-\alpha)[1+\delta(p-1)]} a_{k} \leq 1
$$

which readily yields

$$
\sum_{k=j+p}^{\infty} k ! a_{k} \leq \frac{(p-\alpha)[1+\delta(p-1)](j+p) !}{\frac{(\lambda+p)_{j}(c)_{j}}{j !(a)_{j}}(j+p-\alpha)[1+\delta(j+p-1)]} .
$$

Now, by differentiating both sides of (1.1) m times, we obtain

$$
\begin{aligned}
f^{(m)}(z)= & \frac{p !}{(p-m) !} z^{p-m}-\sum_{k=j+p}^{\infty} \frac{k !}{(k-m) !} a_{k} z^{k-m} \\
& (k \geq j+p, j, p \in N) .
\end{aligned}
$$

Theorem 2, follows from (3.3) and (3.4).

Finally, it is easy to see that the bounds in (3.1) are at- tained for the function $f(z)$ given by (3.2).

\section{Radii of Close-to-Convexity, Starlikeness and Convexity}

Theorem 3. Let the function $f(z)$ defined by (1.1) be in the class $S C_{n}(j, p, \lambda, \alpha, \delta)$ then

1) $f(z)$ is $p$-valently close-to-convex of order 
$\varphi(0 \leq \varphi<p)$ in $|z|<r_{1}$, where

$$
r_{1}=\inf _{k}\left\{\frac{\frac{(\lambda+p)_{k-p}(c)_{k-p}}{(k-p) !(a)_{k-p}}(k-\alpha)[1+\delta(k-1)]}{(p-\alpha)[1+\delta(p-1)]}\left(\frac{p-\varphi}{k}\right)\right\}^{\frac{1}{k-p}},\left(k \geq j+p, p, j \in N, n \in N_{0}\right),
$$

2) $f(z)$ is p-valently starlike of order $\phi(0 \leq \varphi<p)$ in $|z|<r_{2}$, where

$$
r_{2}=\inf _{k}\left\{\frac{\frac{(\lambda+p)_{k-p}(c)_{k-p}}{(k-p) !(a)_{k-p}}(k-\alpha)[1+\delta(k-1)]}{(p-\alpha)[1+\delta(p-1)]}\left(\frac{p-\varphi}{k-\varphi}\right)\right\}^{\frac{1}{k-p}},(k \geq j+p p, j \in N)
$$

3) $f(z)$ is p-valently convex of order $\varphi(0 \leq \varphi<p)$ in $|z|<r_{3}$, where

$$
r_{3}=\inf _{k}\left\{\frac{\frac{(\lambda+p)_{k-p}(c)_{k-p}}{(k-p) !(a)_{k-p}}(k-\alpha)[1+\delta(k-1)]}{(p-\alpha)[1+\delta(p-1)]} \cdot \frac{p(p-\varphi)}{k(k-\varphi)}\right\}^{\frac{1}{k-p}},(k \geq j+p, j, p \in N) .
$$

Each of these results is sharp for the function $f(z)$ given by (2.7).

Proof. It is sufficient to show that

$$
\begin{gathered}
\left|\frac{f^{\prime}(z)}{z^{p-1}}-\varphi\right| \leq p-\varphi\left(|z|<r_{1} ; 0 \leq \varphi<p, p \in N\right), \\
\left|\frac{z f^{\prime}(z)}{f(z)}-p\right| \leq p-\varphi\left(|z|<r_{2} ; 0 \leq \varphi<p, p \in N\right),
\end{gathered}
$$

and

$$
\left|1+\frac{z f^{\prime \prime}(z)}{f^{\prime}(z)}-p\right| \leq p-\varphi\left(|z|<r_{3} ; 0 \leq \varphi<p, p \in N\right),
$$

for a function $f(z) \in S C_{n}(j, p, \lambda, \alpha, \delta)$ where $r_{1}, r_{2}$ and $r_{3}$ are defined by (4.1) - (4.3) respectively. The details involved are fairly straightforward and may omi- tited.

\section{Modified Hadamard Products}

For the functions $f_{i}(z)(i=1,2)$ defined by

$$
f_{i}(z)=z^{p}-\sum_{k=j+p}^{\infty} a_{k, i} z^{k}\left(a_{k, i} \geq 0 ; i=1,2\right),
$$

we denote by $\left(f_{1} * f_{2}\right)(z)$ the modified Hadamard product (or convolution) of the functions $f_{1}(z)$ and $f_{2}(z)$, defined by

$$
\left(f_{1} * f_{2}\right)(z)=z^{p}-\sum_{k=j+p}^{\infty} a_{k, 1} a_{k, 2} z^{k} .
$$

Theorem 4. Let the functions $f_{i}(z)(i=1,2)$ defined by (5.1) be in the class $S C_{n}(j, p, \lambda, \alpha, \delta)$ then $\left(f_{1} * f_{2}\right)(z) \in S C_{n}(j, p, \lambda, \gamma, \delta)$, where

$$
\gamma=p-\frac{j(p-\alpha)^{2}[1+\delta(p-1)]}{\frac{(\lambda+p)_{j}(c)_{j}}{j !(a)_{j}}(j+p-\alpha)^{2}[1+\delta(j+p-1)]-(p-\alpha)^{2}[1+\delta(p-1)]} .
$$

The result is sharp for the functions $f_{i}(z)(i=1,2)$ given by

$$
f_{i}(z)=z^{p}-\frac{(p-\alpha)[1+\delta(p-1)]}{\frac{(\lambda+p)_{j}(c)_{j}}{j !(a)_{j}}(j+p-\alpha)[1+\delta(j+p-1)]} z^{j+p},(p, j \in N, i=1,2) .
$$


Proof. Employing the technique used earlier by Schild and Silverman [12], we need to find the largest $\gamma$ such that

$$
\sum_{k=j+p}^{\infty} \frac{\frac{(\lambda+p)_{k-p}(c)_{k-p}}{(k-p) !(a)_{k-p}}(k-\gamma)[1+\delta(k-1)]}{(p-\gamma)[1+\delta(p-1)]} a_{k, 1} a_{k, 2} \leq 1 .
$$

Since $f_{i}(z) \in S C_{n}(j, p, \lambda, \alpha, \delta)(i=1,2)$, we readily see that

$$
\sum_{k=j+p}^{\infty} \frac{\frac{(\lambda+p)_{k-p}(c)_{k-p}}{(k-p) !(a)_{k-p}}(k-\alpha)[1+\delta(k-1)]}{(p-\alpha)[1+\delta(p-1)]} a_{k, i} \leq 1
$$
tain

Therefore, by the Cauchy-Schwarz inequality, we ob-

$$
\frac{(p-\alpha)[1+\delta(p-1)]}{\frac{(\lambda+p)_{k-p}(c)_{k-p}}{(k-p) !(a)_{k-p}}(k-\alpha)[1+\delta(k-1)]} \leq \frac{(p-\gamma)(k-\alpha)}{(p-\alpha)(k-\gamma)},(k \geq j+p, p, j \in N),
$$

It follows from (5.10) that

$$
\gamma \leq p-\frac{(k-p)(p-\alpha)^{2}[1+\delta(p-1)]}{\frac{(\lambda+p)_{k-p}(c)_{k-p}}{(k-p) !(a)_{k-p}}(k-\alpha)^{2}[1+\delta(k-1)]-(p-\alpha)^{2}[1+\delta(p-1)]},(k \geq j+p, j, p \in N) .
$$

Now, defining the function $G(k)$ by

$$
G(k)=p-\frac{(k-p)(p-\alpha)^{2}[1+\delta(p-1)]}{\frac{(\lambda+p)_{k-p}(c)_{k-p}}{(k-p) !(a)_{k-p}}(k-\alpha)^{2}[1+\delta(k-1)]-(p-\alpha)^{2}[1+\delta(p-1)]},(k \geq j+p, j, p \in N) .
$$

We see that $G(k)$ is an increasing function of $k$. Therefore, we conclude that

$$
\gamma \leq G(j+p)=p-\frac{j(p-\alpha)^{2}[1+\delta(p-1)]}{\frac{(\lambda+p)_{j}(c)_{j}}{j !(a)_{j}}(j+p-\alpha)^{2}[1+\delta(j+p-\alpha)]-(p-\alpha)^{2}[1+\delta(p-1)]},
$$

which evidently completes the proof of Theorem 4 .

Remark: Putting 1) $a=p+1, \lambda=1, c=1, \delta=0$ and 2) $a=p+1, \lambda=1, c=1, \delta=1$ in Theorem 4 , we obtain

Corollary 2. Let the functions $f_{i}(z)(i=1,2)$ defined by (5.1) be in the class $T_{j}^{*}(p, \alpha)$. Then $\left(f_{1} * f_{2}\right)(z) \in$ $T_{i}^{*}(p, \gamma)$, where

$$
\gamma=p-\frac{j(p-\alpha)^{2}}{(j+p-\alpha)^{2}-(p-\alpha)^{2}} .
$$

The result is sharp.

Corollary 3. Let the functions $f_{i}(z)(i=1,2)$ defined by (5.1) be in the class $C_{j}(p, \alpha)$. Then $\left(f_{1} * f_{2}\right)(z) \in$ $C_{j}(p, \gamma)$, where

$$
\gamma=p-\frac{j(p-\alpha)^{2} p}{(j+p-\alpha)^{2}(1+j+p-\alpha)-(p-\alpha)^{2} p} .
$$

The result is sharp.

Using arguments similar to those in the proof of 
Theorem 4, we obtain the following result.

Theorem 5. Let the function $f_{1}(z)$ defined by (5.1) be in the class $S C_{n}(j, p, \lambda, \alpha, \delta)$ Suppose also that the function $f_{2}(z)$ defined by (5.1) be in the class $S C_{n}(j, p, \lambda, \tau, \delta)$, Then $\left(f_{1} * f_{2}\right)(z) \in S C_{n}(j, p, \lambda, \zeta, \delta)$, where

$$
\zeta=p-\frac{j(p-\alpha)(p-\tau)[1+\delta(p-1)]}{\frac{(\lambda+p)_{k-p}(c)_{k-p}}{(k-p) !(a)_{k-p}}(j+p-\alpha)(j+p-\tau)[1+\delta(j+p-1)]-\Omega},
$$

and

$$
\Omega=(p-\alpha)(p-\tau)[1+\delta(p-1)] .
$$

The result is the best possible for the functions

$$
f_{1}(z)=z^{p}-\frac{(p-\alpha)[1+\delta(p-1)]}{\frac{(\lambda+p)_{j}(c)_{j}}{j !(a)_{j}}(j+p-\alpha)[1+\delta(j+p-1)]} z^{j+p},(p, j \in N)
$$

and

$$
f_{2}(z)=z^{p} \frac{(p-\tau)[1+\delta(p-1)]}{\frac{(\lambda+p)_{j}(c)_{j}}{j !(a)_{j}}(j+p-\tau)[1+\delta(j+p-1)]} z^{j+p},(p, j \in N) .
$$

Theorem 6. Let the functions $f_{i}(z)(i=1,2, \cdots, m)$ defined by (5.1) be in the class $S C_{n}(j, p, \lambda, \alpha, \delta)$. Then the function

$$
h(z)=z^{p}-\sum_{k=j+p}^{\infty}\left(\sum_{i=1}^{\infty} a_{k, i}^{2}\right) z^{k},
$$

belongs to the class $S C_{n}(j, p, \lambda, \zeta, \delta)$ where

$$
\zeta=p-\frac{j m(p-\alpha)^{2}[1+\delta(p-1)]}{\frac{(\lambda+p)_{j}(c)_{j}}{j !(a)_{j}}(j+p-\alpha)^{2}[1+\delta(j+p-1)]-m(p-\alpha)^{2}[1+\delta(p-1)]} .
$$

The result is sharp for the functions $f_{i}(z)(i=1,2, \cdots, m)$ given by (5.4).

Proof. Noting that

$$
\begin{gathered}
\sum_{k=j+p}^{\infty}\left\{\frac{\frac{(\lambda+p)_{k-p}(c)_{k-p}}{(k-p) !(a)_{k-p}}(k-\alpha)[1+\delta(k-1)]}{(p-\alpha)[1+\delta(p-1)]}\right\}^{2} a_{k, i}^{2} \leq\left\{\sum_{k=j+p}^{\infty} \frac{\frac{(\lambda+p)_{k-p}(c)_{k-p}}{(k-p) !(a)_{k-p}}(k-\alpha)[1+\delta(k-1)]}{(p-\alpha)[1+\delta(p-1)]} a_{k, i}\right\} \leq 1 \\
\left(f_{i}(z) \in S C_{n}(j, p, \lambda, \alpha, \delta)(i=1,2, \cdots, m)\right)
\end{gathered}
$$

we have

$$
\sum_{k=j+p}^{\infty} \frac{1}{m}\left\{\frac{\frac{(\lambda+p)_{k-p}(c)_{k-p}}{(k-p) !(a)_{k-p}}(k-\alpha)[1+\delta(k-1)]}{(p-\alpha)[1+\delta(p-1)]}\right\}^{2}\left(\sum_{i=1}^{m} a_{k, i}^{2}\right) \leq 1 .
$$

Therefore, we have find largest $\zeta$ such that 


$$
\frac{(k-\zeta)}{(p-\zeta)} \leq \frac{\frac{(\lambda+p)_{k-p}(c)_{k-p}}{(k-p) !(a)_{k-p}}(k-\alpha)^{2}[1+\delta(k-1)]}{m(p-\alpha)^{2}[1+\delta(p-1)]}(k \geq j+p, p, j \in N),
$$

that is, that

$$
\zeta \leq p-\frac{m(k-p)(p-\alpha)^{2}[1+\delta(p-1)]}{\frac{(\lambda+p)_{k-p}(c)_{k-p}}{(k-p) !(a)_{k-p}}(k-\alpha)^{2}[1+\delta(k-1)]-m(p-\alpha)^{2}[1+\delta(p-1)]},(k \geq j+p, p, j \in N) .
$$

Now, defining the function $\psi(k)$ by

$$
\psi(k)=p-\frac{m(k-p)(p-\alpha)^{2}[1+\delta(p-1)]}{\frac{(\lambda+p)_{k-p}(c)_{k-p}}{(k-p) !(a)_{k-p}}(k-\alpha)^{2}[1+\delta(k-1)]-m(p-\alpha)^{2}[1+\delta(p-1)]},(k \geq j+p, p, j \in N)
$$

we observe that $\psi(k)$ is an increasing function of $k$. We thus conclude that

$$
\zeta \leq \psi(j+p)=p-\frac{m j(p-\alpha)^{2}[1+\delta(p-1)]}{\frac{(\lambda+p)_{j}(c)_{j}}{j !(a)_{j}}(j+p-\alpha)^{2}[1+\delta(j+p-1)]-m(p-\alpha)^{2}[1+\delta(p-1)]},
$$

which completes the proof of Theorem 6 .

\section{Applications of Fractional Calculus}

Various operators of fractional calculus (that is, fractional integral and fractional derivatives) have been studied in (cf., e.g., [9,10,13-15]; see also the various references cited therein).

For our present investigation, we recall the following definitions.

Definition 1. The fractional integral of order $\mu$ is defined, for a function $f(z)$, by

$$
D_{z}^{-\mu} f(z)=\frac{1}{\Gamma(\mu)} \int_{0}^{z} \frac{f(\zeta)}{(z-\zeta)^{1-\mu}} \mathrm{d} \zeta \quad(\mu>0)
$$

where the function $f(z)$ is analytic in a simply-connected domain of the complex z-plane containing the origin and the multiplicity of $(z-\zeta)^{\mu-1}$ is removed by requiring $\log (z-\zeta)$ to be real when $(z-\zeta)>0$.
Definition 2. The fractional derivative of order $\mu$ is defined, for a function $f(z)$, by

$$
D_{z}^{\mu} f(z)=\frac{1}{\Gamma(1-\mu)} \int_{0}^{z} \frac{f(\zeta)}{(z-\zeta)^{\mu}} \mathrm{d} \zeta \quad(0 \leq \mu<1),
$$

where the function $f(z)$ is constrained, and the multiplicity of $(z-\zeta)^{-\mu}$ is removed, as in Definition 1.

Definition 3. Under the hypotheses of Definition 2, the fractional derivative of order $n+\mu$ is defined, for a function $f(z)$, by

$$
D_{z}^{n+\mu} f(z)=\frac{d^{n}}{d z^{n}}\left\{D_{z}^{\mu} f(z)\right\} \quad\left(0 \leq \mu<1, n \in N_{0}\right) .
$$

In this section, we shall investigate the growth and distortion properties of functions in the class $S C_{n}(j, p, \lambda, \alpha, \delta)$ involving the operators $J_{c, p}$ and $D_{z}^{\mu}$. In order to derive our results, we need the following Lemma given by Chen et al. [14].

Lemma 1 (see [14]). Let the function $f(z)$ defined by (1.1). Then

$$
D_{z}^{\mu}\left\{J_{c, p} f(z)\right\}=\frac{\Gamma(p+1)}{\Gamma(p+1-\mu)} z^{p-\mu}-\sum_{k=j+p}^{\infty} \frac{(c+p) \Gamma(k+1)}{(c+k) \Gamma(k+1-\mu)} a_{k} z^{k-\mu},(\mu \in R ; c>-p, p, j \in N)
$$

and

$$
J_{c, p}\left(D_{z}^{\mu}\{f(z)\}\right)=\frac{(c+p) \Gamma(p+1)}{(c+p-\mu) \Gamma(p+1-\mu)} z^{p-\mu}-\sum_{k=j+p}^{\infty} \frac{(c+p) \Gamma(k+1)}{(c+k-\mu) \Gamma(k+1-\mu)} a_{k} z^{k-\mu}, \quad(\mu \in R ; c>-p, p, j \in N)
$$

provided that no zeros appear in the denominators in (6.4) and (6.5). 
Theorem 7. Let the function $f(z)$ defined by (1.1) be in the class $S C_{n}(j, p, \lambda, \alpha, \delta)$. Then

$$
\left|D_{z}^{-\mu}\left\{\left(J_{c, p} f\right)(z)\right\}\right| \geq\left\{\begin{array}{c}
\frac{\Gamma(p+1)}{\Gamma(p+1-\mu)}-\frac{(c+p) \Gamma(j+p+1)(p-\alpha)[1+\delta(p-1)]}{(c+j+p) \Gamma(j+p+1+\mu) \frac{(\lambda+p))_{j}(c)}{j !(a)_{j}}(j+p-\alpha)[1+\delta(j+p-1)]}|z|^{j} \\
(z \in U ; 0 \leq \alpha<p ; 0 \leq \delta \leq 1 ; \mu>0 ; c>-p, p, j \in N)
\end{array}\right\}|z|^{p+\mu},
$$

and

$$
\left|D_{z}^{-\mu}\left\{\left(J_{c, p} f\right)(z)\right\}\right| \leq\left\{\begin{array}{c}
\frac{\Gamma(p+1)}{\Gamma(p+1+\mu)}+\frac{(c+p) \Gamma(j+p+1)(p-\alpha)[1+\delta(p-1)]}{(c+j+p) \Gamma(j+p+1+\mu) \frac{(\lambda+p)_{j}(c)_{j}}{j !(a)_{j}}(j+p-\alpha)[1+\delta(j+p-1)]}|z|^{j} \\
(z \in U ; 0 \leq \alpha<p ; 0 \leq \delta \leq 1 ; \mu>0 ; c>-p, p, j \in N) .
\end{array}|z|^{p+\mu},\right.
$$

Each of the assertion (6.6) and (6.7) is sharp.

Proof. In view of Theorem 1, we have

$$
\frac{\frac{(\lambda+p)_{j}(c)_{j}}{j !(a)_{j}}(j+p-\alpha)[1+\delta(j+p-1)]}{(p-\alpha)[1+\delta(p-1)]} \sum_{k=j+p}^{\infty} a_{k} \leq \sum_{k=j+p}^{\infty} \frac{\frac{(\lambda+p)_{k-p}(c)_{k-p}}{(k-p) !(a)_{k-p}}(j+p-\alpha)[1+\delta(k-1)]}{(p-\alpha)[1+\delta(p-1)]} a_{k} \leq 1,
$$

which readily yields

$$
\sum_{k=j+p}^{\infty} a_{k} \leq \frac{(p-\alpha)[1+\delta(p-1)]}{\frac{(\lambda+p)_{j}(c)_{j}}{j !(a)_{j}}(j+p-\alpha)[1+\delta(j+p-1)]} .
$$

Consider the function $f(z)$ defined in $U$ by

$$
\begin{aligned}
F(z) & =\frac{\Gamma(p+1+\mu)}{\Gamma(p+1)} z^{-\mu} D_{z}^{-\mu}\left\{\left(J_{c, p} f\right)(z)\right\}=z^{p}-\sum_{k=j+p}^{\infty} \frac{(c+p) \Gamma(k+1) \Gamma(p+1+\mu)}{(c+k) \Gamma(k+1+\mu) \Gamma(p+1)} a_{k} z^{k} \\
& =z^{p}-\sum_{k=j+p}^{\infty} \phi(k) a_{k} z^{k}(z \in U),
\end{aligned}
$$

where

$$
\phi(k)=\frac{(c+p) \Gamma(k+1) \Gamma(p+1+\mu)}{(c+k) \Gamma(k+1+\mu) \Gamma(p+1)}(k \geq j+p, p, j \in N, \mu>0) .
$$

Since $\phi(k)$ is a decreasing function of $k$ when $\mu>0$, we get

$$
0<\phi(k) \leq \phi(j+p)=\frac{(c+p) \Gamma(j+p+1) \Gamma(p+1+\mu)}{(c+j+p) \Gamma(j+p+1+\mu) \Gamma(p+1)}(c>-p, p, j \in N, \mu>0) .
$$

Thus, by using (6.9) and (6.11), we deduce that

$$
\begin{aligned}
|F(z)| \geq|z|^{p}-\phi(j+p)|z|^{j+p} \sum_{k=j+p}^{\infty} a_{k} \\
\geq|z|^{p}-\frac{(c+p) \Gamma(j+p+1) \Gamma(p+1+\mu)(p-\alpha)[1+\delta(p-1)]}{(c+j+p) \Gamma(j+p+1+\mu) \Gamma(p+1) \frac{(\lambda+p)_{j}(c)_{j}}{j !(a)_{j}}(j+p-\alpha)[1+\delta(j+p-1)]}|z|^{j+p},
\end{aligned}
$$


and

$$
\begin{aligned}
|F(z)| \leq|z|^{p}+\phi(j+p)|z|^{j+p} \sum_{k=j+p}^{\infty} a_{k} \\
\leq|z|^{p}+\frac{(c+p) \Gamma(j+p+1) \Gamma(p+1+\mu)(p-\alpha)[1+\delta(p-1)]}{(c+j+p) \Gamma(j+p+1+\mu) \Gamma(p+1) \frac{(\lambda+p)_{j}(c)_{j}}{j !(a)_{j}}(j+p-\alpha)[1+\delta(j+p-1)]}|z|^{j+p},
\end{aligned}
$$

which yield the inequalities (6.6) and (6.7) of Theorem 7. tion $f(z)$ given by The equalities in (6.6) and (6.7) are attained for the func-

$$
D_{z}^{-\mu}\left\{\left(J_{c, p} f\right)(z)\right\}=\left\{\frac{\Gamma(p+1)}{\Gamma(p+1+\mu)}-\frac{(c+p)(p-\alpha) \Gamma(j+p+1)[1+\delta(p-1)]}{(c+j+p) \Gamma(j+p+1+\mu) \frac{(\lambda+p)_{j}(c)_{j}}{j !(a)_{j}}(j+p-\alpha)[1+\delta(j+p-1)]}|z|^{j}\right\} z^{p+\mu}
$$

or, equivalently, by

$$
\left(J_{c, p} f\right)(z)=z^{p}-\frac{(c+p)(p-\alpha)[1+\delta(p-1)]}{(c+j+p) \Gamma(j+p+1+\mu) \frac{(\lambda+p)_{j}(c)_{j}}{j !(a)_{j}}(j+p-\alpha)[1+\delta(j+p-1)]} z^{j+p} .
$$

Thus we complete the proof of Theorem 7.

Using arguments similar to those in the proof of be in the class $S C_{n}(j, p, \lambda, \alpha, \delta)$. Then

Theorem 7, we obtain the following result.

$$
\left|D_{z}^{\mu}\left\{\left(J_{c, p} f\right)(z)\right\}\right| \geq\left\{\begin{array}{c}
\left.\frac{\Gamma(p+1)}{\Gamma(p+1+\mu)}-\frac{(c+p) \Gamma(j+p+1)(p-\alpha)[1+\delta(p-1)]}{(c+j+p) \Gamma(j+p+1-\mu) \frac{(\lambda+p)_{j}(c)_{j}}{j !(a)_{j}}(j+p-\alpha)[1+\delta(j+p-1)]}|z|^{j}\right\}|z|^{p-\mu}, \\
(z \in U ; 0 \leq \alpha<p, 0 \leq \delta \leq 1,0 \leq \mu<1, c>-p, j, p \in N),
\end{array}\right.
$$

and

$$
\left|D_{z}^{\mu}\left\{\left(J_{c, p} f\right)(z)\right\}\right| \leq\left\{\frac{\Gamma(p+1)}{\Gamma(p+1-\mu)}+\frac{(c+p) \Gamma(j+p+1)(p-\alpha)[1+\delta(p-1)]}{(c+j+p) \Gamma(j+p+1-\mu) \frac{(\lambda+p)_{j}(c)_{j}}{j !(a)_{j}}(j+p-\alpha)[1+\delta(j+p-1)]}|z|^{j}\right\}|z|^{p-\mu},
$$

Each of the assertions (6.14) and (6.15) is sharp.

\section{References}

[1] P. L. Duren, “Univalent functions,” In: Grundlehen der
Mathematischen Wissenschaften, Vol. 259, SpringerVerlag, New York, 1983.

[2] A. W. Goodman, "Univalent Functions, Vols. I and II," Polygonal Publishing House, Washington, 1983.

[3] S. Owa, “The Quasi-Hadamard Products of Certain Ana- 
lytic Functions,” In: H. M. Srivastava and S. Owa, Eds., Current Topics in Analytic Function Theory, World Scientific Publishing Company, Singapore, 1992, pp. 234251.

[4] Z.-G. Wang, R. Aghalary, M. Darus and R. W. Ibrahim, "Some Properties of Certain Multivalent Analytic Functions Involving the Cho-Kwon-Srivastava Operator," Journal of Mathematical and Computer Modelling, Vol. 49, No. 9-10, 2009, pp. 1969-1984.

[5] N. E. Cho, O. S. Kwon and H. M. Srivastava, "Inclusion Relationships and Argument Properties for Certain Subclasses of Multivalent Functions Associated with a Family of Linear Operators," Journal of Mathematical Analysis and Applications, Vol. 292, No. 2, 2004, pp. 470-483. doi:10.1016/j.jmaa.2003.12.026

[6] R. Yamakawa, "Certain Subclasses of p-Valently Starlike Functions with Negative Coefficients," In: H. M. Srivastava and S. Owa, Eds., Current Topics in Analytic Function Theory, World Scientific Publishing Company, Singapore, 1992, pp. 393-402.

[7] S. D. Bernardi, "Convex and Starlike Univalent Functions," Transactions of the American Mathematical Society, Vol. 135, 1969, pp. 429-446. doi:10.1090/S0002-9947-1969-0232920-2

[8] A. E. Livingston, "On the Radius of Univalence of Certain Analytic Functions," Proceedings of the American Mathematical Society, Vol. 17, No. 2, 1966, pp. 352-357. doi:10.1090/S0002-9939-1966-0188423-X
[9] H. M. Srivastava and S. Owa (Eds.), "Current Topics in Analytic Function Theory,” World Scientific Publishing Company, Singapore, 1992.

[10] S. Owa, “On Distortion Theorems. I,” Kyungpook Mathematical Journal, Vol. 18, 1978, pp. 55-59.

[11] H. M. Srivastava and M. K. Aouf, "A Certain Fractional Derivative Operator and Its Applications to a New Class of Analytic and Multivalent Functions with Negative Coefficients. I and II," Journal of Mathematical Analysis and Applications, Vol. 171, No. 1, 1992, pp. 1-13. doi:10.1006/jmaa.1995.1197

[12] A. Schild and H. Silverman, "Convolutions of Univalent Functions with Negative Coefficients," Annales Universitatis Mariae Curie-Sklodowska Section A, Vol. 29, 1975, pp. 99-107.

[13] O. Altintas, H. Irmak and H. M. Srivastava, "Fractional Calculus and Certain Starlike Functions with Negative Coefficients," Computers and Mathematics with Applications, Vol. 30, No. 2, 1995, pp. 9-15. doi:10.1016/0898-1221(95)00073-8

[14] M.-P. Chen, H. Irmak and H. M. Srivastava, "Some Families of Multivalently Analytic Functions with Negative Coefficients," Journal of Mathematical Analysis and Applications, Vol. 214, No. 2, 1997, pp. 674-690. doi:10.1006/jmaa.1997.5615

[15] H. M. Srivastava and S. Owa (Eds.), "Univalent Functions, Fractional Calculus, and Their Applications,” Ellis Horwood Limited, Chichester, 1989. 